Zu diese $\mathrm{Z}$ weck werden die Benzocoeroxnniumsalze zuerst mit $Z$ ink und Eisessig zu den Bepzocoeroxenolen reducirt, mit Wasser gefallt, abfiltrirt und auf einem Thonteller etwas getrocknet. Hierauf wurden sie in dem doppelten bis dreifachen Volumen Jodwasserstoffsäure vom. Sdp. $126^{\circ}$ gelöst und 2-3 Stunden gekocht. Das Ende der Reaction bann daran erkannt werden, dass eine herausgenommene Probe in alkoholischem Kali sich wicht mehr mit rother Farbe löst. Ist die Reaction zu Ende, so fällt man mit Wasser, filtrirt und behandelt den. Niederschlag mit schwach ammoniakalischem Wasser bis zum Verschwinden der sauren Reaction; dann wird getrocknet und schliesslich. mit Benzol extrahirt. Das Benzocoeroxen ist leicht löslich in Alkohol und Benzol, etwas weniger in Ligroïn. Bei der Reduction entsteht jedoch nur sehr wenig. Die Hauptmenge ist ein in Benzol unlöslicher Körper, der sich in covcentrirter Schwefelsäure tief violett löst und durch Verdünnen in violetten Flocken ausfällt. Theilweise mit gelbgrüner Fluorescenz in Chloroform und theilweise mit rothvioletten Farbe in Nitrobenzol löslich.

Genf, Universitätslaboratorium.

357. A. Windsus: Ueber Cholesterin. VII. [Ans der medic. Abtheilung des Universitătslaboratoriums zu Freiburg i. B.] (Eingegangen am 18. Juni 1906.)

Bei der Oxydation des Cholesterins mit Chromsäure in Eisessig. lösung haben Mauthner und Suida') drei neutrale Oxydationsproducte aufgefanden, das $\alpha$-Oxycholestenol, das Oxycholestenon und das $\mathbf{O x y -}$ cholestendiol ${ }^{8}$ ). Auf Grund der bisher festgestellten Thatsachen lässt. sich etwa das Folgende über diese Körper aussagen. Das $\alpha$-Oxycholestenol, $\mathrm{C}_{27} \mathrm{H}_{42} \mathrm{O}_{2}$, ist ein ungesättigter, secundärer Alkohol; ob aber seine Hydroxylgruppe und seine Doppelbindung noch dieselben sind, wie die ursprünglich im Cholesterin vorbandenen, erscheint zweifelhaft; er liefert ein Monoacetat; die Function des zweiten Sauerstoffatoms ist unbekannt. Das Oxycholestenon ist das dem Oxycholestenol entsprechende Keton; es bildet ein sebr charakteristisches Phenylhydrazon. Das Oxycholestendiol, $\mathrm{C}_{27} \mathrm{H}_{42} \mathrm{O}_{3}$, ist wahrscheinlich ein gesättigter Körper, der sich vom Oxycholestenon durch den Mehrgehalt von einem.

1) Monatsh. für Chem. 17, 5i9 [1896].

2) Die für diese Verbindungen gewåhlten Namen entsprechen nicht mehr . rollständig unserer erweiterten Kenntniss über ihre chemicche Constitution. 
Molekül Wasser unterscheidet. Durch wasserentziehende Mittel gebt er glatt in Oxycholestenon über. Es ist wahrscheinlich, dass das Molekül Waster an die doppelte Bindung des Oxycholestenons angelagert ist.

Im Anschluss an die grundlegenden Arbeiten ron Mauthner und Suida hat van Oordt') einige Versuche angestellt. Er hat gefunden, dass Oxycbolestendiol mittels Salpeteroäure, Oxycholestenol und Oxycholestendiol auch durch Kaliumperunanganat und Schwefeleäure aus dem Cholesterin entetehen. An das Oxycholestunon hat er ein Molekül Blausäure addiren kōnnen; ab diese Anlagerung an die Doppelbindung oder an die Ketogruppe stattfindet, ist unentechieden. Das Cyanid zeigt die $\mathbf{E}$ gentbümlichkeit, bereits beim Umkrystallioiren aus Alkohol seine Blausäure wieder abzugeben und sich in Oxycholestenon zurückzuverwandeln'). Die Addition ion Blausãure stellt al:o einen schon bei gewöhnlicher Temperatur reversiblen Vorgang dar, bei welchem Bindungen zwischen Kohlenstoffatomen gebildet bezw. gelöst werden. Solche Processe sind bekanntlich nicht häufig ${ }^{3}$. In alkoholischer Lösung bei Gegenwart von Salzsăure ist bingegen das Cyanid, das übrigens der Verseifung einen erbeblichen Widerstand entgegensetzt, besıändig und spaltet keine Blausäure ab. Von weiteren Beobachtungen van Oordt's sei angeführt, dass Oxycholestendiol beim Behandeln mit alkoholischer Salzeäure nicht in Oxycholestenon, $\mathrm{C}_{27} \mathrm{H}_{4}, \mathrm{O}_{2}$, sondern in eine Substanz $\mathrm{C}_{84} \mathrm{H}_{44} \mathrm{O}_{*}$ übergeht, in der nach der Zeisel'schen Methode eine Aethoxylgruppe nachgewiesen werden kann. Dieser Körper, der poch Ketoneigenschafien besitzt, entstebt auch direct aus dew Oxycbolestenon mit Alkohol und Salzsäure und ist als dessen Aetbyläther aufzufassen ${ }^{4}$ )

Vor einiger'Zeit habe ich die Untersuchung des Oxycholestenons wieder aufgenommen, in der Neinung, dass es für den weiteren Fortschritt in der Cholesterin Chemie ernünscht sei, zunächst über den Bildungsmechanismus der ersten Oxydationsprodu'te in's Klare zu kommen.

Oxydation des Cholesterin3: $20 \mathrm{~g}$ Cholesterin wurden in $180 \mathrm{ccm}$ Benzol und $20 \mathrm{ccm}$ Eisessig gelōst, unter Küblung mit $160 \mathrm{ccrn}$

1) Ueber Cholesterin, Inaugural-Dissertation, Freiburg i. B. 1901.

2) A. Windaus, Ueber Cholesterin, Habilitationssebrift, Freiburg i. B. 1903 , S. 36 .

3) Vergl. den analogen Zerfall des $\mu$-Oxy- $\alpha$ Cyanobenzylanilins in Blausäure and Benzyliden $p$-Aminophenol. (F. Sachs und Goldmann, diese Berichte 35, 3323 [(1902]).

4) Winda s, Habilitationsschrift, S. 33. 
der von Kiliani ${ }^{1}$ ) angegebenen (hromsäuremischung versetat und dann (i Stunden geschüttelt. Das Reactionsprodact wurde in der üblichen (schon wiederholt beschriebenen) Weise in saure und geutrale Producte getrennt. Die neutralen, die ca. $70 \mathrm{pCt}$. ausmachen, warden aach dem Verfabien ron Mauthner and $S$ uida") auf Oxycholestenon verarbeitet, von welchem $5-6 \mathrm{~g}$ in reinem Zustand isolirt wurden.

Die Säuren: Dio eingedampften sauren Producte wurden in einzelnen Portionen mit verechiedenen Lobungemitteln stehen gelassen. In dem mit Aceton versetzten Gemisch schieden sich hierbei im Verlauf mehrerer Wochen Krystalle aus, die filtrirt, mit Aceton gewascben und wiederholt aus Eisessig umkrystallisirt wurden. Schmp. $290^{\circ}$, sebr schwer löslich in den üblichen Lösungsmitteln. Bei băufiger Wiederbolang der Oxydation gelang es nunmebr regelmässig, durch Lösen der sauren Producte in Aceton und Impfen mit diesen Krystallen in Laufe eives Tages Krystallabscheidung $2 u$ erzielen; doch betrug die Ausbeute nur 3-4 $\mathrm{pCt}$. vom angewandten Cholesterin

$0.2584 \mathrm{~g}$ Sbst.: $0.7110 \mathrm{~g} \mathrm{CO}, 0.2362 \mathrm{~g} \mathrm{H}_{2} \mathrm{O}$.

$$
\begin{array}{ll}
\mathrm{C}_{27} \mathrm{H}_{46} \mathrm{O}_{4} \text {. Ber. C 75.05, } \mathrm{H} 10.22 . \\
\text { Gef. } 74.94, ~ 10.26 .
\end{array}
$$

Titration: $0.4097 \mathrm{~g}$ Sbst. verbrauchten $18.7 \mathrm{~cm} / 10-n$. Natronlauge. Aequivalentgewicht für $\mathrm{C}_{87} \mathrm{H}_{44} \mathrm{O}_{4}$ (zweibasisch). Ber. 2!3. Gef. 219.

Eine Säure von der gleichen Formel und den gleichen Eigenscbaften haben bereits $\mathrm{Diels}$ und Abderhalden bei der Oxydation des (bolestering mit unterbromigeaurem Kalium erhalten ${ }^{2}$ ). Von der Identität der beiden Verbindungen habe ich mich auseer dareh directen Vergleich anch dadurch überzeugt, dass ich mein l'sodtuet in den charakteristischen sauren Methyleater übergeführt habe, wekcher den von Diels und Abderbalden. angegebenen Schmp. (1240) zeigte. Die Säure entsteht in der Weise aus dem Cholesterin, dass die cyclisch gebundene Gruppe . $\mathrm{CH}(\mathrm{OH}) \mathrm{CH}_{2}$. unter Bildung zweier Carboxyle aufgesprengt wird.

\section{Ueber Oxycholestenon.}

Die in der Einleitung etwähnte Bildung eines Aethyläthers aus dem Oxycholestenon spricht für das Vorhandensein einer Hydroxylgruppe, die voraussichılich wegen ibrer Beständigkeit gegen Chromsäure tertiärer Natur sein dürfte. Um diese Annabme sicherer zu

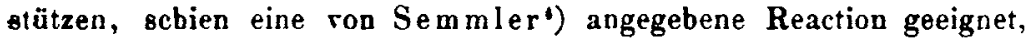

1) Diese Berichte 34, 3564 [1901].

\%) Monatsh. für Chem. 17, 582 [1896].

3) Diese Berichte 36, 3177 [1903]: 37, 3092 [1904].

) Diese Berichte 27, 25:0 [15.91]; 33, 7i6 [1ऽ00;. 
nach welcher nur tertiäres Hydroxyl beim Erhitzen mit Zinkstaub im Rohr durch Wasserstoff ersetzt wird.

Reduction ${ }^{1}$ ): $5 \mathrm{~g}$ Oxycholestenon wurden mit $10 \mathrm{~g}$ Zinkstaub 2 Stunden anf $180^{\circ}$ erhitzt, das braun gefärbte Reactionsproduct mit Benzol extrahirt und nach dem Abdunsten des Lösungsmittels mit Aether stehen gelassen. Dieser setzte allmählich eine Kruste von Krystallen ab, die filtrirt und wiederbolt aus Eisessig unter Zusatz ron Blutkohle umkrystallisirt wurden. Schmp. 170-171'. Ausbeute kaum 10 pCt.

0.1986 g Sbst.: $0.5894 \mathrm{~g} \mathrm{CO}, 0.1996 \mathrm{~g} \mathrm{H}_{2} \mathrm{O}$.

$$
\begin{aligned}
& \mathrm{C}_{27} \mathrm{H}_{42} \mathrm{O}_{2} \text {. Ber. C 81.32, H } 10.65 \text {. } \\
& \text { Gef. \80.94, \11.24. }
\end{aligned}
$$

Ein Körper ron demselben Schmelzpunkt und derselben Formel ist früher auf einem ganz anderen Wege (über Nitrocholesterin) von mir aufgefunden und als gesättigtes Diketon charakterisirt worden, das Cholestandion ${ }^{2}$ ), und thatsäcblich ist, wie eine sorgfältige Untersuchung ergab (Miscbschmelzpunkt, Dioxim), das Product aus Oxycholestenon mit dem Cholestandion identisch. Ein Vergleich der Formeln für Oxycholestenon $\left(\mathrm{C}_{27} \mathrm{H}_{40} \mathrm{O}_{2}\right)$ und für Cholestandion $\left(\mathrm{C}_{27} \mathrm{H}_{42} \mathrm{O}_{2}\right)$. zeigt, dass beim Erbitzen mit Zinkstaub ein Ersatz einer Hydroxylg' uppe durch Wasserstoff nicht stattgefunden hat, und dass demgemäss das Oxycholestenon wabrscheinlich keine tertiäre Hydroxylgruppe entbält. Vielmehr ist höchst wahrscbeinlich eine Addition von 2 Wasserstoffatomen eingetreten. $\mathrm{Da}$ aber das Oxycholestenon möglicherweise auch die Formel $\mathrm{C}_{27} \mathrm{H}_{42} \mathrm{O}_{2}$, also dieselbe wie das Cholestandion, besitzt, könnte es sich vielleicht anch nur um eine Umlagerung innerhalb des Moleküls handeln; dann aber sollte Oxycholestenon voraussicbtlich auch durch blosses Erbitzen in Cholestandion überführbar sein. Dies ist aber nicht der Fall, selbst wenn Zinkoxyd als Katalysator ${ }^{3}$ ) zugesetzt wird. Aus dem stark verfärbten Reactionsproduct liess sich nur unrerändertes Oxycholestenon isoliren.

Der Uebergang von Oxycholestenon in Chole:tandion beim Erhitzen mit Zinkstaub verläuft so wenig glatt, dass er zu Constitutionsbestimwungen nicht verwerthbar ist. Unter anderen Reactionsbedingungen gelingt indessen die quantitative Ueberfübrung der einen Substanz in die andere:

1) Die Versuche mussten vielfach variirt werden, bis es gelang, krystallisirte Reductionsproducte zu erbalten. Auf die Mittheilung der nothwendigen. Vorversuche ist stets verzichtet.

3) Diese Berichte 36, 3755 [1903].

3) s, hierzu: Ipatiew und Leon towitsch, diese Berichte 36, 2016 [1903]- 
5 g Oxycholestenon in $125 \mathrm{ccm}$ Eisessig werden mit $5 \mathrm{~g}$ Zinkstaub and $10 \mathrm{ccm}$ Wasser 4 Stunden am Rückflusskühler gekocht, die farblose Iösung mit viel Wasser versetzt, das ausgefallene Product abfiltrirt und aus Alkohol umkrystallisirt. Schmelzpunkt und Verhalten gegen Lösungs mittel wie beim Cholestandion. Zur ganz sicheren Feststellung der Identität wurde es in die Cholestanondisäure ${ }^{1}$ ) und weiter in deren sehr charakteristischen Methylester (Schmp. 1130) verwandelt.

Durch Kochen mit Eisessig und essigsaurem Zink wird dagegen das Oxycholestenon nicht verändert, es handelt sich also thatsächlich um eine Reduction.

Der glatte Uebergang des Oxycholestenons in das Cholestandion ${ }^{2}$ ) lässt keinen $Z$ weifel daran, dass ersteres ein ungesättigtes Diketon ist; seine beiden Ketogruppen müssen ebenso wie diejenigen des Cholestandions in zwei verschiedenen bydrirten Ringen stehen ${ }^{3}$ ). Da seine Doppelbindung so ausserordentlich leicht reducirt wird, so ist es nicht $\mathrm{zw}$ eifelhaft, dass sie sich in $\alpha, \beta$-Stellung mindestens $z a$ einer der beiden Ketogruppen befindet.

Die Enolgruppe des Oxycholestenons: Die Bildung des Oxycholestenonäthyläthers ${ }^{4}$ ) ist augenscheinlich nur dadurch zu erklären, dass die eine der beiden Ketogruppen des Oxycholestenons in der Enolform reagirt ${ }^{5}$ ). Dass es sich bei dem Aethyläther thatsächlich am einen Enoläther handelt, wird noch durch andere Beobachtungen gestützt: Bei dem Versuch, den Aethyläther mit Zinkstaub und Eisessig zu reduciren, fand ich nämlich, dass bierbei ledig. lich Cholestandion entstand, dass also ausser der Reduction auch Verseifung eingetreten war. Ich habe mich dann überzeugt, dass beim Kochen des Oxycholestenonäthyläthers mit Eisessig und Zinkacetat glatt eine Zurückverwandelung in Oxycholestenon stattfindet. Der Aetbyläther ist also schon durch Erwärmen mit Essigsäure verseifbar,

1) Diese Berichte 36, 3756 [1903].

2) Für die Darstellung des Cholestandions ist der neue Weg über das Oxycholestenon einfacher als der frühere über das Nitrocholesterin; er liefert indessen etwas geringere Ausbeuten.

3) Eine Umlagerung beim Uebergang des Oxycholestenons in Cholestandion ist sehr wenig wahrscheinlich. Immerhin sei an den Befund von Erlenmeyer erinnert (diese Berichte 36, 2527 [1903]), dass die Cinnamylameisensāure mit der Gruppe.CH:CH.CO.CO. bei der Reduction in Benzoylpropionsäure mit der Gruppe $. \mathrm{CO} \cdot \mathrm{CH}_{2} \cdot \mathrm{CH}_{2} . \mathrm{CO}$. übergeführt werden kann. Ebenso könnte vielleicht ein $\alpha, \beta$-ungesättigtes Orthodiketon .CH:CH.CO.CO . $z \mathfrak{u}$ einem gesăttigten $\gamma$-Diketon reducirt werden.

4) Auch ein Benzyläther vom Schmp. $132^{\circ}$ ist darstellbar.

5) Cholestenon und Cholestandion liefern beim Kochen mit alkoholischer Salzsăure keine Aethyläther.

Berichte d. D. chem. Gesellschaft. Jahrg. XXXIX. 
ein für Aether scheinbar sehr abnormes Verbalten. Indessen ist, wie Knorri) kürzlich bervorgehoben und durch Beispiele erläutert hat, die grosse Unbeständigkeit gerade für Enoläther besonders charakteristisch.

Für das Vorhandensein einer Enolgruppe im Oxycholestenon sprechen auch folgende Beobachtungen. Wird Oxycholestenon in ätherischer Lösung mit 10-proc. oder verdünnterer Kalilange geschüttelt, so wirkt die Lauge nicht ein. Verwendet man aber eine 20-proc. oder stärkere Kalilauge, so bildet sich ein intensiv gelbgefärbtes Kaliumsalz ${ }^{2}$ ) des Oxycholestenons, das sich unlöslich abscheidet. In frischem Zustande wird dieses Kaliumsalz durcb Wasser in seine Bestandtheile (Kali und Oxycholestenon) zerlegt. In essigsaurer Lösung liefert es mit Phenylhydrazin das charakteristische Oxycholestenonphenylhydrazon. Lässt man es aber im trocknen Zustand im Vacuum stehen, so erfährt es im Laufe einiger Tage eine bemerkenswerthe Verwandlung. Mit Eisessig und Phenylhydrazon liefert es kein Hydrazon mehr, in Wasser ist es vollständig löslicb geworden, und aus dieser Lösung wird durch Mineralsäuren eine organische Säure ausgefällt. Das Kaliumsalz des Oxycholestenons hat sich also beim Aufbewahren in das Salz einer Säure umgelagert; letztere ist bisher nicht untersucht.

Die Bromide des Oxycholestenons: Die nahe Verwandtschaft $z$ wischen Oxycholestenon und Cholestandion fand noch eine weitere überraschende Bestätigung. Mautbner und Suida ${ }^{3}$ ) haben an Oxycholestenon $1 \mathrm{Mol}$. Brom addiren können und dabei ein krystallisirtes Dibromid erhalten, das bei $167-168^{\circ}$ schmolz. Ich habe aus dem Cholestandion ein Dibromsubstitutionsproduct ${ }^{4}$ ) dargestellt, das sich bei ca. $165^{\circ}$ zersetzte. Die Formeln der beiden Verbindungen sind dieselben; die geringe Verschiedenheit im Zersetzungspunkt kommt nicht in Betracht, im Verhalten zu LösungsmitteIn besteht kein Unterschied. Um die Identität der beiden Dibromide za beweisen, hat Hr. Dr. F. M. Jäger in Zaandam (Holland) die Güte gebabt, sie krystallographisch zu vergleichen. Die vollständig durchgeführten Messungen, für die ich Hrn. Dr. Jäger zu grossem Danke verpflichtet bin, haben nun mit voller Sicherheit ergeben, dass die beiden Verbindungen identisch sind.

1) Diese Berichte 39, 1410 [1906].

2) Nach O. Widman ist die gelbe Farbe für die Oxyvinylsalze einigermaassen charakteristisch. Diese Berichte 35, 1158 [1902].

3) Monatsh. für Chem. 17, 588 [1896].

3) Diese Berichte 37, 2031 [1904]. 
Dieser Identitätsnachweis findet seine Bestätigung auch darin, dass sich die beiden Dibromide bei weiterer Einwirkung ron Brom in dasselbe Tribromsubstitutionsproduct überführen lassen: $5 \mathrm{~g}$ Cholestandion in $50 \mathrm{ccm}$ Chloroform wurden mit $10 \mathrm{~g} \mathrm{Brom} \mathrm{in} 100 \mathrm{ccm}$ Chloroform und einigen Kryställchen Jod versetzt und 2 Tage stehen gelassen. Dann wurde die Lösung durch Schütteln mit wässriger, schwefliger Säure von überschüssigem Halogen befreit, im Vacuum eingedampft und der Rückstand, der aus einem Gemisch von Dibromid und Tribromid besteht, durch Auskochen mit Alkohol vom Dibromid befreit. Das zurückbleibende Tribromid wurde dann aus heissem Aceton umkrystallisirt, aus dem es in langen Nadeln vom Schmp. $195^{0}$ ausfällt.

$0.1876 \mathrm{~g}$ Sbst.: $0.3523 \mathrm{~g} \mathrm{CO}_{2}, 0.1102 \mathrm{~g} \mathrm{H}_{2} \mathrm{O} .-0.1650 \mathrm{~g}$ Sbst.: $0.1471 \mathrm{~g}$ $\mathrm{Ag} \mathrm{Br}$.

$$
\begin{aligned}
& \mathrm{C}_{27} \mathrm{H}_{39} \mathrm{O}_{2} \mathrm{Br}_{3} . \quad \text { Ber. C 51.01, } \mathrm{H} \text { 6.18, Br 37.77. } \\
& \text { Gef. 》51.22, ॠ6.57, » } 37.94 \text {. }
\end{aligned}
$$

Bei entsprechender Behandlung ergab Oxycholestenon in sehr guter Ausbeute dasselbe Derivat, das bei der Analyse stimmende Werthe lieferte. - Bei der Reduction des Dibrom- oder TribromCholestandions mit Zinkstaub und Eisessig oder Alkohol wird Cholestandion leicht zurückgebildet.

Die beiden Bromatome im Oxycholestenondibromid dürften in 1.2-Stellung zu einander stehen, da sie ja an die Doppelbindung des Oxycholestenons addirt sind. Andererseits sollte aber jedes Bromatom auch in $\alpha$-Stellung zu einer Ketogruppe stehen, da bei der Bromirung von Ketonen die Bromatome an die dem Carbonyl benachbarten Kohlenstoffatome zu treten pflegen. Diesen beiden Bedingungen genügt für das Dibromid nur eine Formulirung mit der Gruppe .CO.CHBr.CHBr.CO., die wir darum als wahrscheinlich annehmen dürfen; das Oxycholestenon wäre dann ein ungesättigtes $\gamma$-Diketon mit der Gruppe $\mathrm{CO} . \mathrm{CH}: \mathrm{CH} . \mathrm{CO} .^{1}$ ), das Cholestandion das entsprechende gesättigte Diketon.

Die 1.4-Stellung der Carbonylreste: Um die 1.4-Stellung der beiden Ketograppen im Cholestandion und im Oxycholestenon mit grösserer Sicherheit nachzuweisen, habe ich eine Reihe von Versuchen ausgeführt.

Verhalten des Cholestandions zu Ammoniak: Bekanntlich geben $\gamma$-Diketone mit Ammoniak Pyrrolderivate. $2 \mathrm{~g}$ Cholestandion

1) Zn derselben Vorstellung über die gegenseitige Lage der Ketogruppen kommt man übrigens anch dann, wenn man für die Enolform des Oxycholestenons die Formulirung $\mathrm{CH}: \mathrm{C}(\mathrm{OH}) \mathrm{CH}: \mathrm{CH}$ annimmt und eine Addition der Bromatome in 1.4-Stellung för möglich hält. 
wurden mit $15 \mathrm{ccm}$ 10-proc. alkoholischem Ammoniak 6 Stunden im Rohr auf $120^{\circ}$ erhitzt. In der Röhre befand sich eine geringe Menge einer gelbrothen, krystallinischen Masse, die nach dem Abgiessen der überstehenden Lösung wiederholt aus Chloroform und Alkobol umkrystallisirt wurde. Hieraus fällt sie in feinen, weissen Nadeln heraus, die erst über $300^{\circ}$ unter Zersetzung schmelzen und Stickstoff enthalten. Ansbeute ca. $8 \mathrm{pCt}$.

Die neue Substanz ist leicht löslich in Chloroform, fast unlöslich in Aethylalkohol und Methylalkohol.

$0.1356 \mathrm{~g}$ Sbst.: $0.4066 \mathrm{~g} \mathrm{CO}_{2}, 0.1391 \mathrm{~g} \mathrm{H}_{2} \mathrm{O} .-0.1922 \mathrm{~g}$ Sbst.: $3.7 \mathrm{ccm}$ N $\left(18^{0}, 741 \mathrm{~mm}\right)$.

$$
\begin{aligned}
& \mathrm{C}_{54} \mathrm{H}_{85} \mathrm{O}_{3} \mathrm{~N} \text { (?). Ber. } \mathrm{C} 81.44, \mathrm{H} 10.76, \mathrm{~N} 1.77 \text {. } \\
& \text { Gef. » 81.78, 》 11.47, 》2.16. }
\end{aligned}
$$

Molekulargewichtsbestimmung: $0.191 \mathrm{~g}$ Sbst.: $10 \mathrm{~g}$ Naphtalin, $0.17^{0} \mathrm{Er}-$ höhung.

$$
\mathrm{C}_{54} \mathrm{H}_{85} \mathrm{O}_{3} \mathrm{~N} \text { (?). Ber. M 795. Gef. M } 782 \text {. }
$$

Analyse und Molekulargewichtsbestimmung zeigen, dass die Verbindung nicht die erwartete Zusammensetzung (eines Pyrrolderivates) besitzt, sondern dimolekular ist. Wahrscheinlich haben 2 Mol. Diketon mit 1 Mol. Ammoniak unter Wasseraustritt reagirt.

Einwirkung von Hydrazin auf Cholestandion: Da der Versuch mit Ammoniak kein einwandsfreies Resultat ergeben hatte, habe ich das Cholestandion mit Hydrazin umgesetzt in der Hoffnung, zu einem Pyridazin- bezw. Dihydropyridazin-Derivat zu gelangen ${ }^{2}$ ). $3 \mathrm{~g}$ Cholestandion wurden mit $100 \mathrm{ccm}$ Alkohol und $2 \mathrm{ccm} 50$-proc. Hydrazinhydrat 6 Stunden gekocht, die Lösung mit Wasser versetzt, das ausgefällte Product abfiltrirt, auf Thon getrocknet, aus BenzolMethylalkohol umkrystallisirt. Hieraus scheidet es sich in sechsseitigen, regelmässigen Blättchen ab, die bei ca. $188^{\circ}$ zusammensintern. Die neue Substanz enthält Stickstoff, sie ist sehr schwer löslich in Aethylalkohol und Petroläther, leicht löslich in Chloroform und Benzol. Die Ausbeute betrug ca. $70 \mathrm{pCt}$.

$0.1798 \mathrm{~g}$ Sbst. (bei $105^{0}$ getrocknet): $0.5413 \mathrm{~g} \mathrm{CO}_{2}, 0.1843 \mathrm{~g} \mathrm{H}_{2} \mathrm{O}$. $0.1154 \mathrm{~g}$ Sbst.: $7.2 \mathrm{ccm} \mathrm{N}\left(14^{0}, 739 \mathrm{~mm}\right)$.

$$
\begin{aligned}
& \mathrm{C}_{27} \mathrm{H}_{42} \mathrm{~N}_{2} \text {. Ber. C 82.15, H 10.73, N 7.12. } \\
& \text { Gef. 》 82.11, 》 11.47, 》 6.94. }
\end{aligned}
$$

Wie die Analyse beweist, hat also 1 Mol. Diketon mit $1 \mathrm{Mol}$. Hydrazin unter Austritt von 2 Mol. Wasser reagirt. Dieses Verhalten zeigen aber, soweit mir bekannt ist, nur $\beta$ - and $\gamma$-Diketone. In

1) Siehe hierzu Paal und Schultze, diese Berichte 35, 168 [19027 Paal und Dencks, diese Berichte 36, 491 [1903] etc. 
einem vereinzelten Fall ist es auch an einem $\alpha$-Diketon ${ }^{1}$ ) beobachtet worden. Da nun Cholestandion sicher kein $\alpha_{-}{ }^{2}$ ) und kein $\beta$-Diketon ${ }^{3}$ ) ist, bleibt als einzige Möglichkeit die 1.4-Stellung der beiden Ketogruppen. Die aus den Resultaten der Bromirung abgeleitete Vermuthung ist damit bestätigt.

Einwirkung von Hydrazin auf Oxycholestenon: Schliesslich wurde auch noch Oxycholestenon mit Hydrazin in Reaction gebracht, um zu prüfen, ob auch hierbei ein Pyridazinderivat gebildet wird. Dies war allerdings von vornherein wenig wabrscheinlich, da Paal und Schulze $)$ gezeigt haben, dass Enolformen von Diketonen sich mit Hydrazin nicht umsetzen. Es war demgemäss zu erwarten, dass Oxycholestenon mit Hydrazin gerade so wie mit Phenylhydrazin ${ }^{5}$ ) nur ein Monohydrazon liefern wärde. Dies ist thatsächlich der Fall: $2 \mathrm{~g}$ Oxycholestenon, in $100 \mathrm{ccm}$ Alkohol gelöst, wurden mit $1 \mathrm{ccm}$ 50-procentigem Hydrazinhydrat versetzt und $4 \mathrm{Stdn}$. unter Rückfluss gekocht. Nach dem Abkühlen schied die Lösung in reichlicher Menge gelbgefärbte Krystallblätter ab, die nach wiederholtem Umkrystallisiren bei $160-161^{\circ}$ schmolzen.

$0.1826 \mathrm{~g}$ Sbst. (bei $105^{0}$ getrocknet): $0.5303 \mathrm{~g} \mathrm{CO}_{2}, 0.1823 \mathrm{~g} \mathrm{H}_{2} \mathrm{O}$. 0.1994 g Sbst.: $11.6 \mathrm{ccm} \mathrm{N}\left(19^{0}, 743 \mathrm{~mm}\right)$.

$$
\begin{aligned}
& \mathrm{C}_{27} \mathrm{H}_{42} \mathrm{ON}_{2} \text {. Ber. C 78.95, H 10.31, N } 6.84 \text {. } \\
& \text { Gef. \79.20, 》11.19, "6.51. }
\end{aligned}
$$

Hieraus geht also hervor, dass 1 Mol. Diketon mit 1 Mol. Hy. drazin unter Austritt von nur $1 \mathrm{Mol}$. Wasser reagirt hat.

\section{Einige weitere Derivate des Oxycholestenons.}

Oxycholestenon und o-Phenylendiamin: Dieser Versuch wurde auf Grund folgender Erwägungen angestellt: Bei der Oxydation des Cholesterins mit Chromsäure entsteht neben Oxycholestenon

1) Curtius und Thun, Journ. für prakt. Chem. 44, 175.

2) Da die beiden Ketogruppen des Cholestandions in zwei $\nabla$ rschiedenen hydrirten Ringen stehen, ist die 1.2-Stellung der Carbonylreste structurell unmöglich.

3) Cholestandion löst sich nicht in Alkalien und giebt ein normales Dioxim. Bei der Oxydation liefert es eine Ketodicarbonsäure mit der gleichen Anzahl Kohlenstoffatomen (die Cholestanondisăure). Letztere müsste, wenn Cholestandion ein $\beta$-Diketon wäre, eine $\alpha$ - oder $\beta$-Ketosäure sein; das erstere ist unmöglich, da die Ketogruppe der Säure cyclisch gebunden ist; das zweite ist ausgeschlossen, da die Cholestanondisäure beim Erbitzen mit Aetzkali selbst auf $200^{\circ}$ beständig ist.

4) Diese Berichte 36, 2386 [1903].

5) Mauthner und Suida, Monatsh. für Chem. 17, 585 [1896]. 
die Säure $\mathrm{C}_{97} \mathrm{H}_{44} \mathrm{O}_{4}$ (s. oben). Wäre Oxycholestenon nun ein $\mathrm{Z}_{w i} \mathrm{i}-$ schenproduct bei der Bildung dieser Säure, so müsste es ein o-Diketon mit cyclisch gebundenen Carbonylgruppen sein; denn nur ein solches könnte bei der Oxydation eine carbonylfreie Dicarbonsäure mit der gleichen Anzahl Kohlenstoffatomen liefern. Nun habe ich zwar bei der Oxydation des Oxycholestenons mit Chromsäure die Säure $\mathrm{C}_{27} \mathrm{H}_{44} \mathrm{O}_{4}$ nicht erhalten können; und dies macht es wahrscheinlich, dass das Oxycholestenon einem in anderer Richtung verlanfenden Oxydationsprocess seine Entstehung verdankt und kein $Z$ wischenproduct bei der Bildang der Säure $\mathrm{C}_{27} \mathrm{H}_{44} \mathrm{O}_{4}$ darstellt. Immerhin habe ich das Verhalten des Oxycholestenons auch gegen o-Phenylendiamin prüfen wollen, um die Annahme eines 1.2 Diketons noch sicherer abweisen zu können ${ }^{1}$ ).

$3 \mathrm{~g}$ Oxycholestenon wurden mit $1.5 \mathrm{~g}$ o-Phenylendiamin $15 \mathrm{Mi}-$ nuten auf $150^{\circ}$ erhitzt. Unter lebhaftem Aufschäumen schmilat die Masse zusammen. Sie wurde in $40 \mathrm{ccm}$ heissem Essigäther gelöst und schied sich nach dem Erkalten in ziegelrothen Blättchen ab, die einige Male aus Essigäther umkrystallisirt wurden. Schmp. $158-159^{\circ}$, schwer löslich in Alkohol und Essigäther, leicht in Chloroform, Aether, Benzol.

$0.2006 \mathrm{~g}$ Sbst.: $0.5998 \mathrm{~g} \mathrm{CO}_{2}, 0.1763 \mathrm{~g} \mathrm{H}_{2} 0 .-0.2018 \mathrm{~g}$ Sbst.: $0.6021 \mathrm{~g}$ $\mathrm{CO}_{2}, 0.1750 \mathrm{~g} \mathrm{H}_{2} \mathrm{O}$ - $-0.1998 \mathrm{~g}$ Sbst.: $10.1 \mathrm{~cm} \mathrm{~N}\left(20^{\circ}, 745 \mathrm{~mm}\right)$.

$$
\begin{aligned}
& \mathrm{C}_{33} \mathrm{H}_{46} \mathrm{ON}_{2} \text {. Ber. C 81.41, } \quad \text { H 9.52, } \quad \text { N 5.77. } \\
& \text { Gef. » } 81.55,81.37, » 9.83,9.70, \gg 5.64 \text {. }
\end{aligned}
$$

Die Substanz enthält also auch nach dem Trocknen bei $105^{\circ}$ noch 1 Mol. Wasser mebr als ein Chinoxalinderivat ${ }^{2}$ ). Weiterhin unterscheidet sie sich in typischer Weise von einem Chinoxalinderivat dadurch, dass sie mit Essigsäureanhydrid acetylirbar ist und hierbei ein in feinen, gelben Nadeln krystallisirendes Product vom Schmp. $148^{\circ}$ liefert. Damit ist die Möglichkeit, dass es sich um ein Chinoxalinderivat handeln könnte, zurückgewiesen; und demgegenüber fällt es nicbt in's Gewicht, dass sich das neue Derivat ähnlich wie viele Chinoxalinderivate mit prachtroll parpurrother Farbe in concentrirter Schwefelsäure oder alkoholischer Salzsäure auflöst.

$$
\begin{gathered}
\text { Oxycholestenon + Essigsäureanhydrid + concentrirte } \\
\text { Schwefelsäure. }
\end{gathered}
$$

Während Oxycholestenon nach Mauthner und Suida ${ }^{3}$ gegen Essigsäureanhydrid indifferent ist, liefert es mit Essigsäureanhydrid

1) S. Anmerkung 3 auf S. 2253.

2) S. hierzu Wedekind und Weisswange, diese Berichte 39, 1634 [1906].

3) Mopatsh. für Chem. 17, 586 . 
und Schwefelsäure ein schwefelhaltiges Product, das sehr merkwürdige Eigenschaften besitzt: $5 \mathrm{~g}$ Oxycholestenon wurden mit einem auf $0^{0}$ abgekühlten Gemisch von $30 \mathrm{~g}$ Essigsäareanhydrid und $5 \mathrm{~g}$ concentrirter Schwefelsäure übergossen; beim Umschwenken löst sich das Oxycholestenon allmählich auf, die Lösung färbt sich röthlich und dann grün, und scheidet in sebr reichlicher Menge weisse Krystalle ab. Nach einigen Stunden wurden diese abgesaugt, mit etwas Eisessig gewaschen und über Natronkalk im Vacuum getrocknet. Zur vollständigen Reinigung wurden sie in heissem Benzol gelöst und mit Petroläther ausgefällt, wobei sie sich als Filzwerk feiner Nadeln abschieden.

Die so gereinigte Substanz zersetzt sich beim Erhitzen im Schmelzpunktsröbrchen bei etwa $148^{\circ}$ unter Schwarzfärbung. In Wasser löst sie sich spielend leicht auf, unter stark saurer Reaction. Die wässrige Lösung lässt auf Zusatz von Cblorkalium oder Chlornatrium die entsprechenden Alkalisalze in krystallisirter Form ausfallen. Mit Chlorcalcium oder Zinksulfat entstehen in Wasser unlösliche Salze, die sich bemerkenswerther Weise in fast allen organischen Lösungsmitteln auflösen.

Anf Grund der Analyse scheint es, als ob die Subsianz aus dem Oxycholestenon durch einfache Anlagerung von Schwefelsäure hervorginge ${ }^{1}$ ); doch bedarf die Formel noch weiterer Stützen. $\mathrm{BaSO}_{4}$.

$0.2152 \mathrm{~g}$ Sbst.( $0.5126 \mathrm{~g} \mathrm{CO}_{2}, 0.1739 \mathrm{~g} \mathrm{H}_{2} \mathrm{O}$. $-0.2182 \mathrm{~g}$ Shst.: $0.1094 \mathrm{~g}$

$$
\begin{aligned}
& \mathrm{C}_{97} \mathrm{H}_{42} \mathrm{SO}_{6} \text { (?). Ber. C 65.54, H 8.56, S } 6.49 \text {. } \\
& \text { Gef. 》 64.96, 》 9.04, } 6.88 \text {. }
\end{aligned}
$$

Zum Schluss sei kurz erwähnt, dass Oxycholestenon auch mit Anilin und mit Schwefelwasserstoff krystallisirte Derivate liefert; doch habe ich von einer Untersuchung derselben Abstand genommen, weil dies von der Hauptaufgabe - dem Studium des Cholesterins zu weit abführen würde.

\section{Anbang:}

Ueber $\beta$-Oxycholestenolacetat und Cholesterilen.

$\beta$-Oxycholestenolacetat: Bei der Oxydation des Cholesterylacetats entstebt, wie Mauthner und Suida ${ }^{2}$ ) gefunden haben, in der Hauptsache das Acetat einer dem $\alpha$-Oxycholestenol isomeren Substanz, des $\beta$-Oxycholestenols. Da nun, wie die oben mitgetheilten Versuche beweisen, das undefinirte Sauerstoffatom im Oxycholestenon und im

1) S. hierzu Vorlä $\mathrm{uder}$ und Mamme, diese Berichte 36, 1481 [1903].

2) Monatsh. für Chem. 17, 594 [1896]. 
$\alpha$-Oxycholestenol einer Ketogruppe zugehört, so lag die Vermuthung nahe, dass genau dasselbe auch für das $\beta$-Oxycholestenol gelten würde. Dies ist in der That der Fall, wie folgender Versuch beweist:

$2 \mathrm{~g} \beta$-Oxycholestenolacetat, in $100 \mathrm{ccm}$ Alkohol gelöst, wurden mit $0.5 \mathrm{~g}$ Hydroxylaminchlorhydrat und $1 \mathrm{~g}$ Natriumacetat, beide in wenig Wasser gelöst, versetzt und I Stunde unter Räckfluss gekocht. Das mit Wasser ausgefällte Reactionsproduct wurde nach dem Trocknen einige Male aus Benzol Petroläther umkrystallisirt. Hieraus fiel es in feinen, verfilzten Nadeln, die bei $185-186^{\circ}$ schmolzen und sich als stickstoffhaltig erwiesen.

$0.1588 \mathrm{~g}$ Sbst.: $0.4436 \mathrm{~g} \mathrm{CO}_{2}, 0.1472 \mathrm{~g} \mathrm{H}_{2} \mathrm{O}-0.1766 \mathrm{~g}$ Sbst.: $0.4955 \mathrm{~g}$ $\mathrm{CO}_{2}, 0.1691 \mathrm{~g} \mathrm{H}_{2} \mathrm{O} .-02522 \mathrm{~g}$ Sbst.: $7 \mathrm{ccm} \mathrm{N}\left(15^{0}, 725 \mathrm{~mm}\right)$.

$$
\begin{aligned}
& \mathrm{C}_{29} \mathrm{H}_{45} \mathrm{O}_{3} \mathrm{~N} \text {. Ber. C 76.42, } \quad \text { H 9.96, N 3.08. } \\
& \text { Gef. »76,18,76.52, 》10.37, 10.71, 》3.10. }
\end{aligned}
$$

Beim Kochen mit Zinkstaub und Eisessig wird $\beta$-Oxycholestenolacetat nicht verändert, ebenso wenig addirt es Brom, wie bereits Mauthner und Suida ${ }^{1}$ ) für den freien Alkohol angegeben baben.

Cholesterilen: Bei der Wasserabspaltung aus Chulesterin muss entweder ein neuer Ring oder eine $z$ weite Doppelbindung gebildet werden. Je nachdem der erste oder der zweite Fall eintritt, muss also der entstehende Koblenwasserstoff, das Cholesterilen, einfach oder doppelt ungesättigt sein. Mautbuer und Suida haben bei ihren Untersuchungen ${ }^{2}$ ) bereits beobachtet, dass Cholesterilen nur 1 Molekül Brom addirt und daraus geschlossen, dass es nur einfach ungesättigt sei. Demgegenüber kōnnte man auf Grund neuer Erfahrungen darauf hinweisen, dass Doppelbindungen bisweilen inactiv gegen Brom sind, dass insbesondere conjugirte Doppelbindungen . C:C.C:C. nach Thiele ${ }^{3}$ meist nur 1 Molekül Brom glatt aufnehmen. Dass im Cholesterilen ein solches System conjugirter Doppelbindungen vorliegen könnte, erschien nun um so eher plausibel, als ja das Cholesterin nach Diels und Abderhalden ${ }^{4}$ ) einen $\alpha, \beta$-ungesättigten Alkohol darstellen sollte, dessen Gruppe $. \mathrm{CH}: \mathrm{CH}$ $. \mathrm{CH}(\mathrm{OH}) . \mathrm{CH}_{8}$ leicht ein System conjugirter Doppelbindungen liefern konnte. Eine Prüfung dieser Annabme auf experimentellem Wege erschien möglich auf Grund der Beobachtungen von Semmler ${ }^{5}$ ), nach denen Kohlenwasserstoffe mit conjugirten Doppelbindungen bei der Behaudlung mit Natrium und Alkohol glatt zwei Atome Wasserstoff

1) Monatsh. für Chem. 17,595 [1896].

2) Monatsh. für Chem. 17, 37 [1896].

3) Ann. d. Chem. 306, 87 [1899].

4) Diese Berichte 37, 3095 [1904]. 5) Diese Berichte 36, 1033 [1903]. 
in 1.4-Stellang aufuehmen und in einfach ungesättigte Verbindungen übergehen.

$4 \mathrm{~g}$ Cholesterilen wurden in $200 \mathrm{ccm}$ kochendem absoluten Alkohol gelöst und im Verlauf einer Stunde mit $16 \mathrm{~g}$ metallischem Natrium versetzt. Aus der Lösung, die in der üblichen Weise verarbeitet wurde, liess sich nur unverändertes Cholesterilen isoliren. Selbst bei der Reduction in amylalkoholischer Lösung erfolgte kein Angriff des Cbolesterilens. Hieraus folgt, dass im Cholesterilen kein System conjugirter Doppelbindungen vorhanden ist und dass also anch das Verhalten des Cholesterilens kein Argument zu Gunsten der $\alpha, \beta$-Stellung der Doppelbindung im Cholesterin liefert. Vielmehr gewinnt die Annabme von Mathner und Suida, nach der bei der Bildung des Cholesterilens ein neuer Ringschluss stattgefunden habe, sehr an Wahrscheinlichkeit.

Demgegenüber könnte das Verhalten des Cholesterilens gegen Oxydationsmittel zunächst onerwartet erscheinen. Wird nämlich reines Cholesterilen unter genau denselben Bedingungen wie Chlolesterin mit Cbromsäuremischung oxydirt, so entsteht in einer Ausbeute von ca. $5 \mathrm{pCt}$. Oxycholestenon, das in reinem Zustand isolirt und durch seinen Schmelzpunkt und sein charakteristisches Phenylbydrazon identificirt wurde. Zur Deutung dieser Erscheinung konnte man annehmen, dass das Cholesterilen unter dem Einfluss der Schwefelsäure des Chromsäuregemisches in Cholesterin zurückverwandelt würde und erst das Letztere bei der Oxydation Oxycholestenon liefere. Diese Vermuthung scheint aber nicht zutreffend. Wenigstens blieb Cholesterilen, als es in Chloroformlösung 6 Tage mit 10. proc. Schwefelsäure geschüttelt wurde, unverändert und nahm kein Wasser auf.

Es sei schliesslich noch die Frage erörtert, wie man sich den Uebergang des Cbolesterins in Oxycholestenon vorstellen soll. Jedenfalls handelt es sich um eine complicirte und anormale Reaction. Aus den bisherigen Untersuchungen ergiebt sich, dass die im Oxycholestenon enthaltene Doppelbindung nicht der Doppelbindung des Cholesterins entsprechen kann; dass das Oxycholestenon und das Cholestandion $\gamma$-Diketone sind; die eine Ketogruppe geht aus der secundären Hydroxylgruppe des Cholesterins, die andere aus der doppelten Bindung hervor. Beide Ketogruppen stehen in zwei verschiedenen bydrirten Ringen. Aus der Untersuchung des Cholestenons ${ }^{1}$ ) wird es aber wahrscheinlich, dass das Cholesterin die Doppelbindung in einer offenen Kette mit der endständigen Gruppe $\mathrm{CH}: \mathrm{CH}_{2}$ ent-

1) Ein Beweis, dass Cholestenon und Cholesterin dasselbe Kohlenstoffscelett exthalten, wäre sehr erwünscht. 
balte. Bei der Bildung des Oxycholestenols und des Cholestanonols müsste also ein neuer Ringschluss stattfinden.

Man könnte diese Ergebnisse in folgenden schematischen Structurformeln zum Ausdruck bringen:

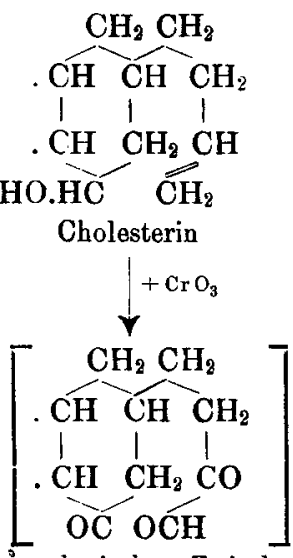

hỹpothetisches Zwischen-

product

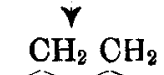

. $\mathrm{CH} \mathrm{CH} \mathrm{CH}_{2}$

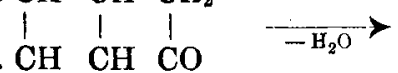

OC CH.OH

Oxycholestendiol

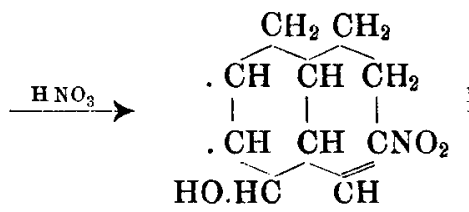

Nitrocholesterin

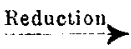

$\mathrm{CH}_{2} \mathrm{CH}_{2}$

CH $\mathrm{CH} \mathrm{CH}_{2}$<smiles>CCC(C=O)C(C)C</smiles>

HO. $\mathrm{HC} \mathrm{CH}_{3}$

Cholestanonol<smiles>[3H]C</smiles>

$\mathrm{CH}_{2} \mathrm{CH}_{2}$

. $\mathrm{CH} \mathrm{CH}_{2} \mathrm{CH}_{2}$

CH $\mathrm{CH} \stackrel{\mathrm{CO}}{ }$

$\mathrm{OC} \mathrm{CH}_{2}$

Cholestandion

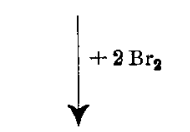

$\mathrm{CH}_{2} \mathrm{CH}_{2}$

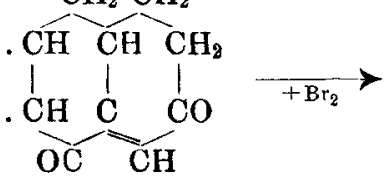

Oxycholestenon

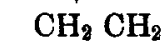

CH $\mathrm{CH} \mathrm{CH}_{2}$<smiles>CC(=O)Br</smiles>

CO CH.Br

Dibromcholestanàion

Diese Formeln enthalten freilich noch manches hypothetisches Element; sie dürften aber geeignet sein, Anhaltspunkte für die Discussion der bisherigen Resultate sowie für die weitere Erforschung des Cholesterins zu liefern. 